\title{
La metodología mixta de investigación aplicada a la perspectiva de género en la prensa escrita
}

\author{
Francisco J. Caro-González ${ }^{1}$ \\ María del Mar García- Gordilloº \\ Ofa Bezunartea-Valencia ${ }^{3}$
}

Recibido: 2013-12-15

Enviado a pares: 2013-12-17
Aprobado por pares: 2014-01-30

Aceptado: 2014-02-03

DOI: 10.5294/pacla.2014.17.3.11

\section{Para citar este artículo / To reference this article / Para citar este artigo}

Caro-González, F.J., García-Gordillo, M.M., Bezunartea-Valencia, O. Septiembre 2014. La metodología mixta de investigación aplicada a la perspectiva de género en la prensa escrita. Palabra Clave 17 (3), 828-853. DOI: 10.5294/pacla.2014.17.3.11

\section{Resumen}

Los métodos mixtos de investigación son cada vez más frecuentes en la investigación en gestión de empresas. Su uso se justifica en tanto que permiten una mayor comprensión de los fenómenos estudiados. El propósito de este artículo es explicar y justificar una metodología que permite responder de forma holística a la cuestión de ¿por qué no leen prensa las mujeres? La metodología, enmarcada en la estrategia del estudio de casos múltiples, integra diferentes técnicas como las entrevistas, las encuestas o el análisis de contenido.

1 Profesor Titular del Departamento de Empresas y Márketing, Facultad de Económicas, Universidad de Sevilla, US, España.fjcaro@us.es.

2 Profesora Contratada Doctora (acreditada de Titular) del Departamento de Periodismo II, Facultad de Comunicación, Universidad de Sevilla, US, España.marggordillo@us.es.

3 Catedrática de Universidad (Profesora Jubilada) del Departamento de Periodismo II, Facultad de Ciencias Sociales y Comunicación, Universidad del País Vasco, UPV, España. ofa@bezunartea.net. 


\section{Palabras clave}

Metodología de investigación, estudios de género, estudio de casos, prensa diaria, investigación cualitativa (Fuente: Tesauro de la Unesco).

\section{Mixed Research Methods Applied to the Gender Perspective in the Press}

\section{Abstract}

Mixed research methods are becoming more and more frequent in research and management consultancy. Their use is justified because they permit a greater understanding of the phenomena being examined. The purpose of this article is to explain and substantiate a methodology that allows for a holistic response to the question: Why don't women read newspapers? Framed by a multiple case study strategy, this methodology combines a variety of techniques, such as interviews, surveys and content analysis.

\section{Keywords}

Research methodology, gender studies, case studies, daily newspapers, qualitative research (Source: UNESCO Thesaurus). 


\section{A metodologia mista de pesquisa aplicada à perspectiva de gênero na imprensa escrita}

\section{Resumo}

Os métodos mistos de pesquisa são cada vez mais frequentes na pesquisa em gestão de empresas. Seu uso se justifica contanto que se permita uma maior compreensão dos fenômenos estudados. O propósito deste artigo é explicar e justificar uma metodologia que permita responder de forma holística à questão de por que as mulheres não leem imprensa. A metodologia, delimitada na estratégia do estudo de casos múltiplos, integra diferentes técnicas como as entrevistas, as enquetes ou a análise de conteúdo.

\section{Palavras-chave}

Metodologia de pesquisa, estudos de gênero, estudos de caso, imprensa diária, pesquisa qualitativa (Fonte: Tesauro da Unesco). 


\section{Introducción}

Los artículos que utilizan metodologías de investigación mixtas cada vez son más usuales en el campo de la administración de empresas (MolinaAzorín, 2012) y tienen un mayor reconocimiento entre los investigadores (Aguinis et al., 2010; Johnson y Onwuegbuzie, 2004; Daft y Lewin, 1990; Lee, 1991).

Las razones por las que se recomienda su uso son variadas: permiten una mayor comprensión de los fenómenos estudiados (Creswell y Plano Clark, 2007), facilitan la aproximación a un problema desde diferentes puntos de vista (Tashakkori y Teddie, 1998) o generan y verifican teorías dentro de una misma investigación (Punch, 2005).

Además, se ha demostrado que las investigaciones multimétodos tienen un mayor impacto que las que utilizan un único método (Molina-Azorín, 2012).

El propósito de este artículo es exponer cómo un grupo multidisciplinar de investigadores da respuesta a una pregunta de investigación amplia y compleja combinando diferentes métodos de investigación. Todo el proceso se encuadra en la estrategia de investigación del estudio de casos múltiples. Esta investigación se ha llevado a cabo en España y en su fase aplicada se probó en la prensa regional andaluza.

El principal reto metodológico del proyecto consiste en integrar los resultados derivados de técnicas de investigación de naturaleza cuantitativa y cualitativa para ofrecer una respuesta coherente a los objetivos.

La cuestión que ha servido de punto de partida y eje para el diseño de la investigación ha sido: ¿Por qué no leen prensa de información general las mujeres?

En este artículo expondremos en primer lugar el germen de la idea de investigación, seguidamente señalaremos las razones que nos llevaron a 
adoptar esta metodología, para a continuación describir los métodos utilizados y, finalmente, demostrar cómo la confluencia de resultados permite dar respuesta a la pregunta planteada.

Este trabajo es por tanto de naturaleza metodológica. No se pretende ofrecer resultados que describan y expliquen las razones por las que las mujeres no leen prensa. Lo que se hace es narrar el proceso de investigación para que pueda servir de modelo, o al menos de inspiración, a otros investigadores que afronten problemas de similar naturaleza. Estamos de acuerdo con Summer et al. (1990), que indicaban que la apertura y la creatividad en la investigación sobre dirección estratégica requieren el uso de diversos métodos de investigación.

\section{Investigación sobre mujeres y medios de comunicación}

Las investigaciones sobre el papel de las mujeres en las empresas de comunicación han adquirido mucha relevancia en los últimos años (Kearney, 2012; Carter y Steiner, 2004; Van Zoonen, 1994).

Desde una óptica empresarial, las investigaciones sobre la relación de las mujeres y los medios de comunicación se pueden clasificar en dos grupos:

- $\quad$ Estudios que analizan la problemática desde la perspectiva interna, es decir, mirando al engranaje de la organización y buscando la respuesta a la desigualdad de género en la cultura, las estructuras, las rutinas y los comportamientos de los actores principales de las empresas (Burke y Stig, 2009; Harp, 2006; Djerf-Pierre, 2007; Limor y Lavie, 2002; Melin-Higgins, 2001; Peters, 2001; Neveu, 2000; Ziamou, 2000).

- Y estudios que se preocupan por conocer el papel de la mujer como objeto y receptoras de la información, cuyas variables de estudio se encuentran ubicadas en el exterior de las organizaciones productoras del mensaje informativo (Batch et al., 2000; Djerf-Pierre, 2007; Peters, 1995). 
Los primeros tratan de comprender el rol desempeñado por las mujeres en el interior de las empresas periodísticas y se centran en temas clave como:

- $\quad$ El techo de cristal: dificultades de las mujeres para acceder al ápice estratégico directivo $y$, por tanto, para tomar decisiones relevantes acerca del producto informativo y la forma de organizar y gestionar el trabajo en las redacciones (Kinnebrock, 2009; Galanaki et al., 2009; Weyer, 2007; Oakley, 2000; Black y Rothman, 1998).

- La segregación horizontal: se analizan los puestos de trabajo y las secciones informativas a las que son relegadas las mujeres al considerarse que son más apropiadas para elaborar un tipo de información denominada 'blanda' o bien porque los hombres copan el territorio de las informaciones públicas y que otorgan mayor prestigio (secciones 'duras') (Hemlinger y Nesbitt, 2006; Djerf-Pierre, 2007; Van Zoonen, 1994).

- Las rutinas informativas: en las que se incide en que la profesión periodística ha sido construida por hombres y para hombres y que, a pesar de la incorporación masiva de las mujeres a las redacciones, todavía no se han implantado medidas de igualdad que favorezcan la conciliación de esta actividad profesional con la vida personal (Murthy y Anita, 2010; Djerf-Pierre, 2007; Melin-Higgins, 2001).

Los tres temas están relacionados y, por ejemplo, unas rutinas de trabajo que dificultan la conciliación de la vida laboral y familiar pueden impedir que las mujeres estén presentes en los procesos de toma de decisiones, o el hecho de trabajar en áreas de menor peso en las redacciones puede dificultar su promoción a los niveles jerárquicos superiores.

Los segundos explican el rol de las mujeres como objetos de la información y como receptoras de la misma. En estos trabajos, normalmente basados en el análisis del producto informativo, se incide en la invisibilización de las mujeres en los medios (Len-Rios et al., 2005), la divulgación de estereotipos de género cuando se informa de ellas (Mateos de Cabo, 
2007; Armstrong y Nelson, 2005) y el uso de un lenguaje sexista (Bach et al., 2000). Por otro lado, y desde la perspectiva más empresarial, se pone de manifiesto el escaso interés que las mujeres muestran por la lectura de prensa general de pago (el caso de la prensa especializada, económica y deportiva, todavía es más extremo), centrándose en que el producto informativo no responde en contenidos o enfoques a las necesidades informativas que ellas tienen. Los empresarios de la comunicación no contemplan como público objetivo a este grupo de la población, por lo que el diseño de los periódicos responde a patrones informativos tradicionales relacionados con el universo masculino (Harp, 2007; Holland, 2004).

Recientes investigaciones han analizado algunas de las relaciones entre los enfoques internos y externos mencionados. De esta manera, Correa y Harp (2011), Kim y Yoon (2009) y Rodgers y Thorson (2003) se detienen en la relación entre la presencia de mujeres en las redacciones y el contenido editorial; Craft y Wanta (2004) y Beam y Di Cicco (2010) se centran, de manera más específica, en la relación entre el porcentaje de mujeres en cargos directivos y el contenido; Armstrong (2004) estudia la relación entre el sexo del reportero y el sexo del protagonista de la noticia, y Zoch y Turk (1998) la relación entre el sexo del redactor y el de las fuentes de información.

Los trabajos hasta aquí reseñados tienen un planteamiento parcial en cuanto al análisis del problema y ofrecen respuestas limitadas al papel de las mujeres en relación con los medios. En este artículo, proponemos una estrategia de investigación ambiciosa que trate de responder de forma global a la problemática de género en las empresas periodísticas. Para ello se utiliza una combinación de metodologías que ayudan a obtener diversos puntos de vista de una misma realidad y nos brindan una visión más amplia y completa. La investigación fue desarrollada en el marco del grupo de investigación Sanimuei, ${ }^{4}$ durante los años 2007-2010, y fue financiado por

4 Sanimuei es el acrónimo del título del proyecto de investigación en el que se enmarca este trabajo: Satisfacción de las Necesidades Informativas de las Mujeres en las Empresas Informativas. 
el Ministerio de Asuntos Sociales y el Instituto de la Mujer. El equipo está compuesto por doctores de diferentes áreas de conocimiento: organización de empresas, marketing, periodismo y publicidad y relaciones públicas. Cada uno de ellos ha aportado sus conocimientos disciplinares, tanto teóricos como metodológicos.

\section{El germen de la investigación}

El grupo Sanimuei parte de la evidencia empírica de que existe una diferencia de hasta 20 puntos entre hombres y mujeres en los porcentajes de audiencia de los diarios de información general españoles. Al mismo tiempo se constata que existen diarios en los que esta diferencia es ligeramente negativa, mientras que en otros es muy positiva. Para tratar explicar esta situación se barajan dos hipótesis relacionadas extraídas de la revisión teórica: 1) las mujeres no leen periódicos porque el producto informativo no responde ni en contenido ni en forma a sus necesidades, y 2) los contenidos y formatos de los periódicos no se ajustan a los gustos y necesidades de las mujeres porque están dirigidos por hombres. En este punto añadimos dos nuevas cuestiones: las mujeres están subrepresentadas en los puestos de toma de decisiones de los periódicos por la segregación vertical que se manifiesta en la existencia del 'techo de cristal' o las mujeres que consiguen superar esas barreras invisibles y alcanzan puestos de poder adoptan los roles de dirección masculinos, por lo que su gestión no se traslada a la oferta informativa de los diarios.

A partir de este razonamiento derivado de la revisión bibliográfica planteamos las siguientes afirmaciones a modo de hipótesis que queremos explorar:

A. La presencia de mujeres directivas en las empresas periodísticas está relacionada con el porcentaje de mujeres lectoras.

B. Las rutinas productivas favorecen una perspectiva 'masculina' de la información.

C. Las mujeres lectoras están más satisfechas con los periódicos en los que las mujeres tiene más peso en las tareas directivas. 
D. Las mujeres ocupan puestos en secciones que se consideran tradicionalmente femeninas (brecha ocupacional).

E. Los contenidos y continente de los diarios no responden a las necesidades informativas de las mujeres.

\section{Objetivo}

El objetivo del presente trabajo es de carácter metodológico. Se trata de exponer y justificar la estrategia de investigación empleada para responder de forma amplia a la pregunta de ¿por qué no leen prensa las mujeres? y las hipótesis que se derivan de ella. Somos conscientes de la dificultad de este reto, dada la diversidad de material recopilado y de técnicas de análisis utilizadas. Queremos compartir esta propuesta novedosa con la comunidad académica en aras de detectar posibles mejoras y soluciones a los interrogantes y procedimientos planteados.

\section{¿Cómo estudiar de forma global la presencia de las mujeres en el periodismo?}

El enfoque global de esta investigación requiere una metodología mixta que permita el acceso a diferentes fuentes de evidencias empíricas, tanto cuantitativas como cualitativas y tanto primarias como secundarias. Por ello se optó por un procedimiento de trabajo de carácter cualitativo (lo que no impide que se utilicen técnicas de investigación cuantitativas), ya que se pretende identificar los conceptos y relaciones que son importantes para las mujeres directivas y sus audiencias. La estrategia de investigación seleccionada es la del estudio de casos múltiples. Un fenómeno tan complejo y multifacético requiere unas herramientas o instrumentos de investigación que cumplan esos mismos requisitos (Weick, 2007, p. 16; Yin, 1984, p. xi).

Para su desarrollo nos basamos en los principios de la grounded theory. La grounded theory (GT) es un procedimiento sistemático de recoger y analizar datos que ayudan a generar teorías cuyas evidencias están basadas en el comportamiento, actos y discursos de las personas investigadas (Castellanos 
et al., 2010, p. 115). En esta metodología, la teoría y los datos interactúan constantemente. No hay trayectoria de investigación rígida ni lineal, se trata de elegir las opciones que mejor respondan a las hipótesis, algunas de las cuales pueden emerger durante la realización de la investigación. Es posible recolectar todo tipo de datos para generar una teoría fundamentada (Strauss y Corbin, 2002; Andréu, García y Pérez, 2007; Andréu y Pérez, 2009).

La GT admite la triangulación de métodos de investigación y de datos para de esta forma tener una comprensión holística de la realidad e incrementar el grado de credibilidad del análisis de la información cualitativa (Denzin, 1978).

Junto al estudio de casos múltiples hemos realizado el análisis de información secundaria de carácter general del sector, que nos ha ayudado a contextualizar los datos obtenidos. Pasamos a describir ambos planteamientos metodológicos: el estudio de casos múltiples y el análisis de datos secundarios.

A. Estudio de casos múltiples

1. Entrevistas semidirigidas

2. Análisis de contenido

3. Análisis de las mujeres lectoras

B. Estudio de datos secundarios

1. Audiencias de diarios por sexos

2. Directivas en medios de comunicación

3. Presencia de las mujeres en asociaciones profesionales

Podemos resumir la triangulación metodológica en la ilustración 1. Los recuadros agrupados en la elipse con línea discontinua son los que se han empleado para el estudio de casos múltiples. 


\section{Ilustración 1 \\ Triangulación metodológica de investigación}

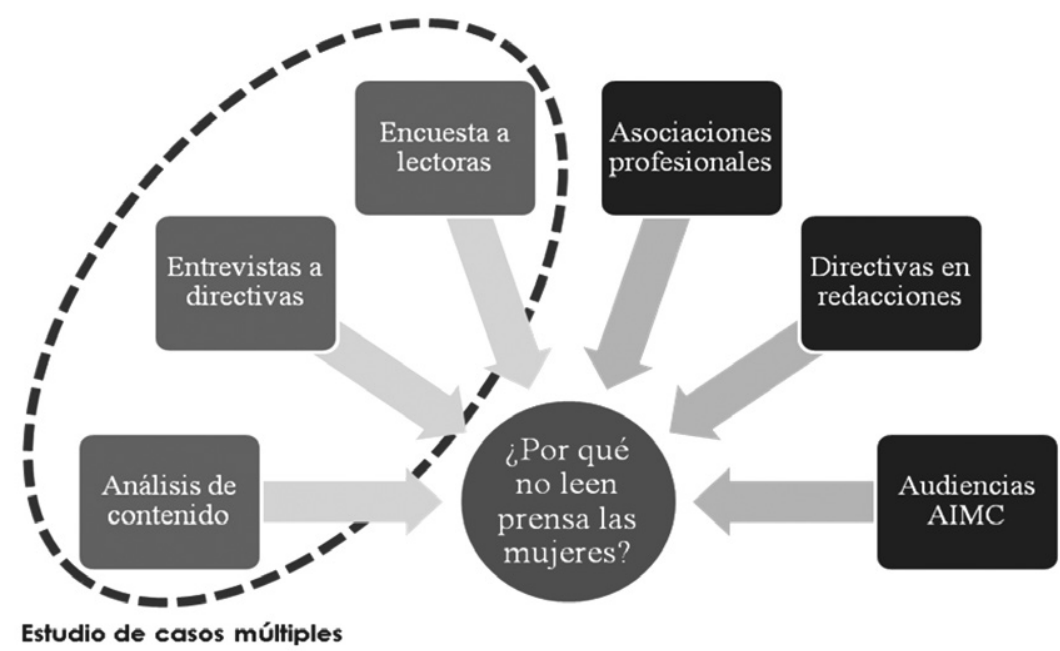

Elaboración propia.

A continuación explicamos con más detalle cada uno de los métodos.

\section{Estudio de casos múltiples}

\section{Selección de los casos}

Debido a que se quiere realizar un estudio comparativo entre casos, se optó por un muestreo teórico de variación máxima (Patton, 1990, pp. 169-181). De entre todos los diarios de la región se seleccionaron los tres que tenían mayor porcentaje de mujeres lectoras y los tres que menos. Para calcular dicho porcentaje se utilizó el promedio de mujeres lectoras durante los años 2000-2009, calculado con los datos ofrecidos por la AIMC (Asociación para la Investigación de Medios de Comunicación).

La investigación se limitó a una región autonómica por cuestiones de accesibilidad a los casos y la ventaja de considerar empresas que actuaban en un entorno socioeconómico homogéneo.

Las empresas se denominaron como A, B y C, en los casos en las que tienen mayor aceptación entre las mujeres, y D, E y F, en los casos de me- 
nor audiencia. Todas las cabeceras son de ámbito provincial y se consideran pequeñas empresas, al tener menos de 50 empleados.

A la hora de analizar la información se llevó a cabo el estudio a tres niveles.

1. Se analizó cada caso de forma individual.

2. Se compararon los resultados de cada grupo de casos (A, B y C, por un lado, y, D, E y F, por otro). En este paso se buscaron similitudes entre las empresas; es lo que se denomina 'replicación literal'.

3. Se compararon los resultados obtenidos en el paso anterior, buscando diferencias entre los dos grupos de caso; es lo que se denomina 'replicación teórica'.

En la ilustración 2 se expone el proceso de análisis seguido con los casos.

\section{Ilustración 2 \\ Planteamiento de estudio de casos múltiples}

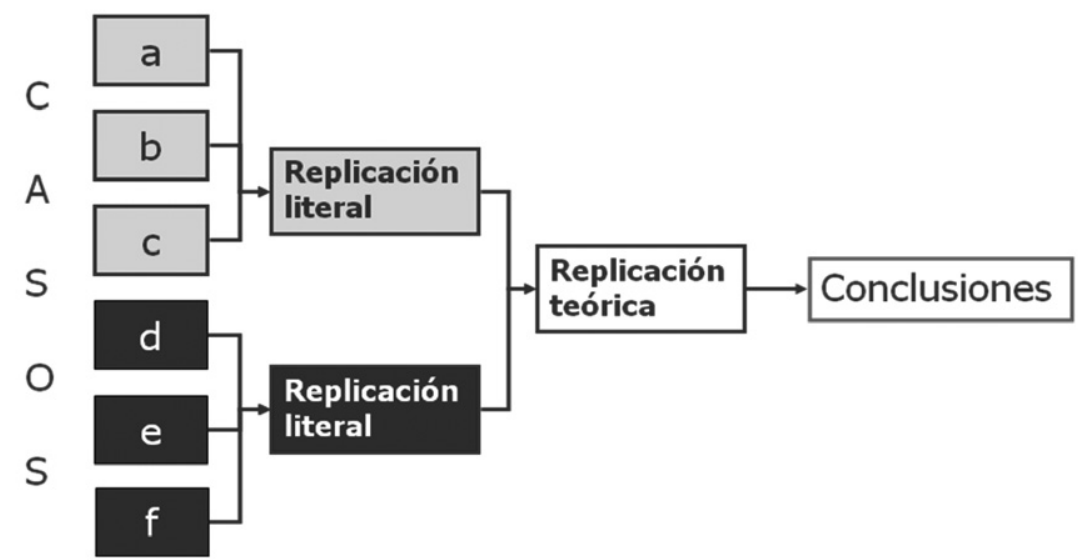

Elaboración propia.

Para cada empresa se recabó la siguiente información:

- $\quad$ Entrevistas semidirigidas.

- Análisis de contenido de los diarios.

- Cuestionario a las mujeres lectoras. 
- $\quad$ Audiencia de los diarios.

- $\quad$ Mujeres en puestos de dirección de cada diario.

Describimos a continuación cómo se utilizó cada una de las fuentes de datos.

\section{Entrevistas semidirigidas}

Se recurrió a informantes clave: mujeres directivas de diferentes niveles jerárquicos pertenecientes a los departamentos de redacción de las empresas seleccionadas.

La pertenencia a los diferentes niveles organizativos ofrece percepciones distintas de la realidad empresarial. Nos centramos en las redacciones, ya que son las profesionales que trabajan en ellas las que tienen más dificultad de conciliar la vida laboral y la familiar, dada la naturaleza del producto periodístico (inmediatez, caducidad, etc.).

Se entrevistó a 12 mujeres directivas pertenecientes a las seis empresas que hemos denominado A, B, C, D, E y F. De ellas, dos son directoras de redacción $(\mathrm{D})$, una es subdirectora $(\mathrm{SD})$, tres son redactoras jefe $(\mathrm{RJ})$ y seis son jefas de sección (JS). Tan sólo cuatro de ellas tienen hijos. Las edades de las entrevistadas oscilan entre los 27 y los 50 años.

\section{Tabla 1}

\section{Directivas entrevistadas}

\begin{tabular}{|c|c|c|c|}
\hline CARGO & EMPRESA & HIJOS & EDAD \\
\hline D1 & A & Sí & $46-50$ \\
\hline JS3 & A & No & $26-30$ \\
\hline JS1 & B & Sí & $46-50$ \\
\hline JS6 & B & No & $36-40$ \\
\hline RJ1 & C & No & $36-40$ \\
\hline RJ2 & C & No & $41-45$ \\
\hline SD & D & No & $41-45$ \\
\hline JS4 & D & No & $26-30$ \\
\hline RJ3 & E & Sí & $46-50$ \\
\hline JS5 & E & No & $26-30$ \\
\hline JS2 & F & Sí & $36-40$ \\
\hline D2 & F & No & $36-40$ \\
\hline
\end{tabular}


Las entrevistas fueron realizadas en las redacciones de los diarios y se aprovechó la visita para tomar nota de información relevante en un cuaderno de campo, donde los investigadores recogían las sensaciones y el lenguaje no verbal de las entrevistadas (Emerson, Fretz y Shaw, 1995). Las entrevistas tuvieron una duración media de 40 minutos.

También se analizó información publicada por las empresas, tanto en las propias páginas de los diarios como en los portales web.

Para el tratamiento de la información recabada se recurrió al programa de análisis de datos cualitativos ATLAS.ti, que presenta ventajas notables en cuanto al ahorro de tiempo, y permite la exploración y codificación intuitiva y sistemática, además de la modelización y visualización analítica (Caro y Díez, 2005). Mediante este software se codificaron las informaciones obtenidas en las entrevistas, diarios de campo y otros documentos de la empresa.

Para reducir la información se siguieron dos tipos de procedimientos: la categorización teórica y la categorización abierta (categorías sugeridas por las fuentes de información).

En la ilustración 3 mostramos las categorías utilizadas agrupadas según el aspecto analizado: la organización, el producto informativo o la audiencia.

\section{Análisis de contenido}

El análisis de contenido es una técnica de investigación destinada a extraer resultados basándose en unos criterios predefinidos. De este método de análisis se destaca, además de la recolección de datos cuantitativos, su capacidad de recoger datos cualitativos, de formular conclusiones según lo puramente estadístico. Además, como todo método científico, se debe prestar a la objetividad y al rigor característico de estas parcelas. De hecho, la efectividad del análisis de contenido ha quedado demostrada en multitud de estudios relacionados con el análisis de la prensa (Agars, 2004).

Para facilitar la comprensión de los pasos seguidos en el proyecto a continuación se desgrana el método paso a paso, a partir de la selección de 


\section{Ilustración 3 \\ Grupos de categorías utilizados}
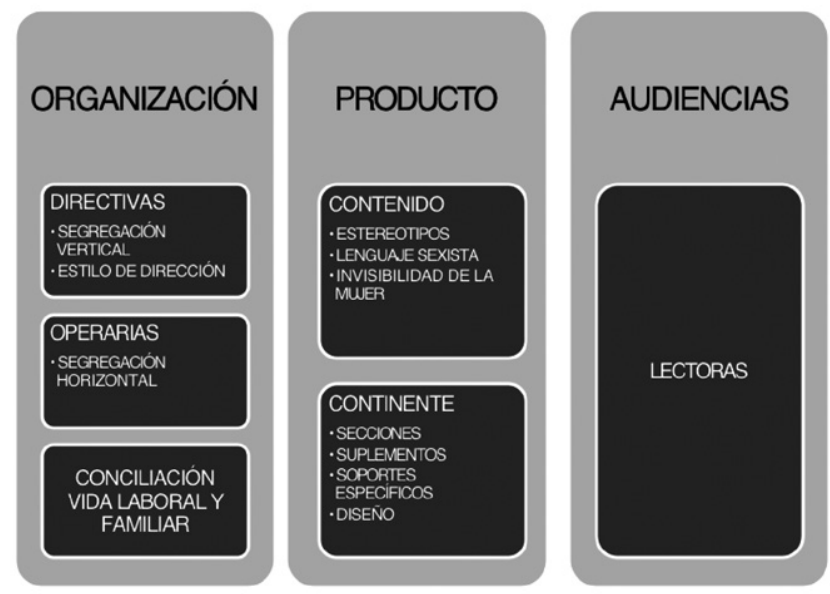

Elaboración propia.

las empresas en función del índice de audiencia de mujeres tal y como hemos mencionado en la elección de los casos de estudio.

Una vez establecido el universo, se seleccionó la muestra de análisis para generar lo que se denomina una semana construida (Krippendorff, 1990; Stempel y Westley, 1989), lo que permite disponer de una muestra amplia no mediatizada por ningún acontecimiento concreto ni por ninguna contingencia. La muestra serían los diarios publicados por cada una de las empresas seleccionadas el lunes de una semana, el martes de la siguiente, el miércoles de la tercera y así hasta cerrar los siete días de la semana. En el caso del proyecto Sanimuei los días fueron:

- lunes 16 de noviembre de 2009,

- martes 24 de noviembre,

- miércoles 2 de diciembre,

- jueves 10 de diciembre,

- viernes 18 de diciembre,

- sábado 26 de diciembre y

- domingo 3 de enero de 2010. 
Después se procedió a la elaboración de las dos fichas de análisis y a la validación de las mismas con una pequeña muestra de textos.

El paso siguiente fue la modificación de la ficha en función de las disfunciones apreciadas en las primeras aplicaciones a los textos.

Siguió una nueva validación de ficha y elaboración de las fichas finales y cierre de las categorías que en ellas se incluyen.

A continuación se procedió a la aplicación de la ficha y cada una de las categorías a cada uno de los textos susceptibles de ser analizados según los criterios definidos en las mismas.

Una vez analizada la muestra y realizadas todas las fichas, se pasó a volcar los resultados de las mismas a una base de datos de Excel.

El paso siguiente fue el traslado de los datos de Excel al programa de análisis SPSS (Statistical Package for the Social Sciences), herramienta muy utilizada en las ciencias sociales, así como en las empresas de investigación de mercado.

Se continuó con la obtención de las tablas de contingencia según los cruces de categorías previamente establecidos.

Por último, se realizó una valoración cualitativa de las tablas de contingencia para la obtención de conclusiones.

\section{Análisis de las mujeres lectoras}

Se recabaron los datos sociodemográficos de las audiencias de los diarios desagregados por sexo que elabora la AIMC (Asociación para la Investigación de Medios de Comunicación). Las categorías tenidas en cuenta fueron: rol familiar, clase social, edad, estado civil, nivel de instrucción, hábitat y audiencia general de medios del año 2009.

Por otra parte se realizó un cuestionario entre las mujeres lectoras de los diferentes diarios. El objetivo principal es obtener información prima- 
ria sobre sus gustos y hábitos de lectura, y de ese modo poder determinar el efecto del contenido del periódico en el índice de lectura de las mujeres.

Entre los posibles tipos de encuesta nos decantamos por la encuesta personal, que se muestra en este caso más adecuada para nuestros objetivos que la telefónica, la postal o la online, ya que la encuestada es seleccionada al pie de quiosco en el momento de la compra del diario y durante la encuesta se pueden aclarar las dudas que les surjan a la hora de interpretar las preguntas.

La población estudiada en nuestro caso son las mujeres lectoras de periódicos que viven en la región. La unidad muestral, que se trata del elemento que va a ser consultado, sería la mujer lectora de periódicos.

El tamaño mínimo de la muestra depende de la población total (la suma de lectoras de todos los periódicos seleccionados):

- N 107000.

Hemos seleccionado una muestra que cumpla los siguientes requisitos:

- Nivel de confianza: $95,50 \%$.

- Error: 5\%.

Con estos datos, el tamaño muestral exigido es:

- $n=398,52$, que ajustada por exceso queda $n=400$.

Para el reparto de la muestra entre los lectores de los diferentes periódicos hemos recurrido a la afijación proporcional, utilizando como ponderación el porcentaje de lectoras de cada periódico en relación con nuestra población. Para eliminar los efectos de los posibles errores a la hora de obtener y tratar la información, hemos ampliado el tamaño muestral un $25 \%$ y también lo hemos repartido proporcionalmente al porcentaje de lectoras de cada periódico. Utilizando estos datos el reparto muestral ha sido el siguiente: 


\section{Tabla 2}

\section{Tamaño muestral definitivo por diario}

\begin{tabular}{|c|c|c|c|c|c|c|}
\hline DIARIO & POBLACIÓN & PESO & MUESTRA & $\begin{array}{c}\text { MUESTRA } \\
\text { AJUSTADA }\end{array}$ & $\begin{array}{c}\text { INCREMENTO } \\
\text { MUESTRA }\end{array}$ & $\begin{array}{c}\text { MUESTRA } \\
\text { TOTAL }\end{array}$ \\
\hline A & 13000 & $12,15 \%$ & 48,59 & 49 & 12 & 61 \\
\hline B & 20000 & $18,69 \%$ & 74,76 & 75 & 19 & 94 \\
\hline C & 29000 & $27,10 \%$ & 108,41 & 108 & 27 & 135 \\
\hline D & 18000 & $16,82 \%$ & 67,28 & 67 & 17 & 84 \\
\hline E & 18000 & $16,82 \%$ & 67,283 & 67 & 17 & 84 \\
\hline F & 9000 & $8,41 \%$ & 33,64 & 34 & 8 & 42 \\
\hline SUMA & 107000 & $100 \%$ & 400 & 400 & 100 & 500 \\
\hline
\end{tabular}

Por último, para garantizar la representatividad y poder extrapolar los resultados del estudio también fueron utilizados elementos probabilísticos. Se sortearon los días y lugares en los que se iba a localizar a las lectoras de estos periódicos. Cada entrevistador proporcionó a la entrevistada un ejemplar del día anterior del mismo periódico que había adquirido, que podría manipular y con relación al cual debería contestar a una serie de preguntas que aparecían en el cuestionario con relación a la lectura del mismo.

Los datos han sido analizados con el software SPSS y se les han aplicado diferentes procesos estadísticos. Realizamos un análisis factorial exploratorio, imputando los valores perdidos con la media de cada variable y aplicando la rotación varimax. Se ha realizado un contraste de medias por grupos. La técnica utilizada ha sido Anova en los casos en que las variables de medida del desempeño eran normales y, en los casos contrarios, se utilizó el test no paramétrico de Kruskal-Wallis. Los resultados nos han permitido identificar diferencias significativas entre los diarios más y menos leídos por las mujeres.

\section{Estudio de datos secundarios}

\subsubsection{Audiencias de diarios por sexos}

Se analizó el porcentaje de audiencia según el sexo de todos los periódicos españoles. Los datos son los elaborados por el EGM (Estudio General de Medios), segregados por sexo desde el año 1999 hasta el 2008. También se calculó el promedio durante ese periodo para establecer un ranking de los 
diarios más leídos (en porcentaje) y los menos leídos por mujeres. En función de que los porcentajes de mujeres lectoras estuviesen por encima de la media, se catalogaron las diferentes empresas como $\mathrm{Aa}$ (alta audiencia de mujeres) o $\mathrm{Ab}$ (baja audiencia de mujeres). Estos datos sirvieron para analizar la relación entre audiencia de mujeres y mujeres con cargos directivos en esos diarios.

\section{Directivas en medios de comunicación}

Para obtener los datos de mujeres directivas se empleó la base de datos de la Agenda de la Comunicación del año 2009, publicada por La Moncloa del Gobierno de España.

En dicha agenda se seleccionaron todas las cabeceras nacionales de prensa general diaria, 103 en total. Para cada empresa se extrajeron los cargos gerenciales y se elaboró una hoja de cálculo en la que se registraron nombre, cargo ocupado y sexo de cada directivo. En total se consideraron como válidas 722 entradas.

Los datos fueron tratados utilizando operaciones estadísticas simples que ponen de manifiesto las desigualdades por sexo que existen en los cargos directivos de las empresas periodísticas.

\section{Presencia de las mujeres en asociaciones profesionales}

Esta parte de la investigación pretende aportar información de contexto para conocer si las formas organizativas de las empresas se repiten en sus asociaciones profesionales. La información la extrajimos del listado de asociaciones publicado en internet por la FAPE (Federación de Asociaciones de la Prensa), donde aparecen enlaces a las diferentes asociaciones provinciales. Dado que no todas las asociaciones ofrecían la información en sus páginas, contactamos telefónicamente con todas ellas. De las 47 asociaciones se consiguieron datos de 32, el 68\% de las existentes en España. El número de registros (miembros de las juntas directivas) fue de 321 .

Se elaboró una hoja de cálculo donde se recoge el número de hombres y mujeres que componen las juntas directivas y se identifica el sexo del presidente de las mismas. 
Los datos fueron tratados utilizando operaciones estadísticas simples que descubren la existencia de desigualdades por sexo en estas organizaciones que representan al colectivo profesional de los periodistas.

\section{El camino de las preguntas a las respuestas}

A continuación presentamos un cuadro resumen que explica la adecuación de las técnicas de investigación con los objetivos y las hipótesis planteadas. En él podemos observar con claridad la triangulación de datos y métodos a la hora de dar respuesta a los interrogantes de la investigación:

\section{Tabla 3 \\ Relación entre objetivos y métodos de investigación}

\begin{tabular}{|c|c|c|c|c|c|}
\hline & \multicolumn{3}{|c|}{$\begin{array}{l}\text { A. Estudio de casos múltiples. } \\
\text { Datos primarios }\end{array}$} & \multicolumn{2}{|c|}{$\begin{array}{l}\text { B. Estudio de datos } \\
\text { secundarios }\end{array}$} \\
\hline & Entrevistas & $\begin{array}{l}\text { Análisis } \\
\text { contenido }\end{array}$ & $\begin{array}{c}\text { Encuesta } \\
\text { lectoras }\end{array}$ & $\begin{array}{c}\text { Audiencias } \\
\text { por sexo }\end{array}$ & $\begin{array}{l}\text { Directivas } \\
\text { en medios }\end{array}$ \\
\hline \multicolumn{6}{|l|}{ HIPÓTESIS: } \\
\hline $\begin{array}{l}\text { A. La presencia de mujeres directivas en las empresas } \\
\text { periodísticas está relacionada con el porcentaje de } \\
\text { mujeres lectoras. }\end{array}$ & $\mathrm{X}$ & & $\mathrm{X}$ & $\mathrm{X}$ & $\mathrm{X}$ \\
\hline $\begin{array}{l}\text { B. Las rutinas productivas favorecen una perspectiva } \\
\text { 'masculina' de la información. }\end{array}$ & $\mathrm{X}$ & & & & \\
\hline $\begin{array}{l}\text { C. Las mujeres lectoras aprecian mayor valor en los } \\
\text { periódicos en los que la mujer tiene más peso en las tareas } \\
\text { directivas. }\end{array}$ & $\mathrm{X}$ & $\mathrm{X}$ & $\mathrm{X}$ & $\mathrm{X}$ & $\mathrm{X}$ \\
\hline $\begin{array}{l}\text { D. Las mujeres ocupan puestos en secciones que } \\
\text { se consideran tradicionalmente femeninas (brecha } \\
\text { ocupacional). }\end{array}$ & $\mathrm{X}$ & $\mathrm{X}$ & & & \\
\hline $\begin{array}{l}\text { E. Los contenidos y continentes de los diarios no } \\
\text { responden a las necesidades informativas de las mujeres. }\end{array}$ & $\mathrm{X}$ & $\mathrm{X}$ & $\mathrm{X}$ & & \\
\hline ¿Por qué no leen las mujeres? & $\mathrm{X}$ & $\mathrm{X}$ & $\mathrm{X}$ & $\mathrm{X}$ & $\mathrm{X}$ \\
\hline
\end{tabular}

La metodología múltiple nos ha permitido hacer un barrido completo de los objetivos propuestos que nos han ayudado a contrastar las hipótesis de investigación. Hay técnicas de investigación con un valor indudable, como las entrevistas en profundidad, que nos han ayudado a descubrir matices que facilitan la interpretación de los datos cuantitativos y en ocasiones responder directamente a la afirmación de la hipótesis. 
Unos métodos se complementan con otros y sirven para dotar de validez interna a la investigación, facilitando la contextualización e interpretación de los datos.

Pero todo no ha sido fácil, ya que en muchas ocasiones hemos tenido que relacionar datos cuantitativos con cualitativos y establecer relaciones conceptuales entre ellos. En este sentido, el esfuerzo y conocimiento de los investigadores procedentes de diferentes campos ha sido clave.

\section{Conclusiones}

La investigación de la problemática de género admite diversos enfoques. Lo fundamental a la hora de aproximarse a este tipo de estudios es plantear de forma correcta una pregunta de investigación, y luego no limitar las posibilidades de dar una respuesta integral a la cuestión por la metodología. La complejidad de la realidad y la dificultad de interpretarla exigen en el investigador una apuesta creativa que combine los múltiples recursos de los que dispone. La calidad de la investigación cualitativa depende en gran medida de la complejidad del fenómeno analizado y de la riqueza y abundancia de los datos (Winter, 2000).

Por otra parte, esta estrategia de investigación se enriquece con la formación de grupos de investigadores procedentes de diferentes áreas de investigación. De esta manera, en Sanimuei participan doctores de las áreas de conocimiento de organización de empresas, periodismo, marketing y publicidad. Cada experto contribuye con sus conocimientos teóricos sobre la materia y el dominio de diferentes metodologías. Esta característica incrementa la calidad de la investigación cualitativa al responder a uno de los criterios especificados por Tracy (2010, p. 841), el de la variedad de teorías y datos que permitan poner de manifiesto los matices y la complejidad de la realidad investigada.

Afortunadamente cada día están más aceptados los enfoques holísticos de la investigación, que integran las metodologías cualitativas y cuantitativas, ofreciendo respuestas más globales y complejas que se aproximan más a la comprensión de los fenómenos sociales. 
La información de esta investigación ha aportado muchos datos que aún se están analizando. Cada método empleado ha permitido estudiar un problema concreto de investigación: percepciones de la profesión por parte de las mujeres directivas, segregación vertical, segregación horizontal, perfil de la mujer lectora, características del producto informativo o el nivel de satisfacción de las mujeres con los diarios.

Es decir, los datos por sí solos ya suponen una información relevante, pero si planteamos la investigación de manera que se puedan cruzar e interrelacionar, el fenómeno estudiado queda mejor perfilado gracias a la triangulación de fuentes informativas, investigadores, métodos e incluso enfoques académicos.

La contrastación de hipótesis ha sido positiva en todas ellas menos en la A, claramente negativa, y la $\mathrm{C}$, donde no hemos encontrado suficientes evidencias concluyentes como para considerarla probada o refutada. Es quizás en la metodología empleada para medir esta afirmación donde deberemos afinar un poco más.

\begin{tabular}{|l|c|}
\hline \multicolumn{1}{|c|}{ HIPÓTESIS: } & Confirmación \\
\hline $\begin{array}{l}\text { A. La presencia de mujeres directivas en las empresas periodísticas está relacionada con el porcentaje } \\
\text { de mujeres lectoras. }\end{array}$ & No \\
\hline B. Las rutinas productivas favorecen una perspectiva 'masculina' de la información. & Sí \\
\hline $\begin{array}{l}\text { C. Las mujeres lectoras aprecian mayor valor en los periódicos en los que la mujer tiene más peso en las } \\
\text { tareas directivas. }\end{array}$ & $i$ Sí \\
\hline $\begin{array}{l}\text { D. Las mujeres ocupan puestos en secciones que se consideran tradicionalmente femeninas (brecha } \\
\text { ocupacional). }\end{array}$ & Sí \\
\hline $\begin{array}{l}\text { F. Los contenidos y su forma de presentación están diseñados sin atender a las necesidades informativas } \\
\text { de las mujeres. }\end{array}$ & Sín \\
\hline
\end{tabular}

\section{Referencias}

Agars, M. D. (2004). "Reconsidering the impact of gender stereotypes on the advancement of women in organizations". En: Psychology of Women Quarterly, 35, pp. 103-111.

Andréu, J. y Pérez, A. M. (2009). "Procesos de investigación interactivos sobre sentimientos de identidad en Andalucía mediante teoría fundamentada [57 párrafos]”. En: Forum Qualitative Sozialforschung/ 
Forum: Qualitative Social Research, 10 (2), art. 18. Disponible en: http://nbnresolving. de/urn:nbn:de:0114-fqs0902187 [fecha de consulta: 21 de mayo de 2010].

Andréu, J., García, A. y Pérez, A. M. (2007). “Evolución de la teoría fundamentada como técnica de análisis cualitativo". En: Cuadernos Metodológicos del CIS, 40, pp. 1-205.

Armstrong, C. L. y Nelson, M. R. (2005). "How newspaper sources trigger gender stereotypes”. En: Journalism \& Mass Communication Quarterly, 82 (4), pp. 820-837.

Armstrong, C. L. (2004). "The influence of reporter gender on source selection in newspaper stories”. En: Journalism \& Mass Communication Quarterly, 81 (1), pp. 139-154.

Beam, R. A. y Di Cicco, D. T. (2010). "When Women Run the Newsroom: Management Change, Gender, and the News”. En: Journalism \& Mass Communication Quarterly, 87 (2), pp. 393-411.

Burke, R. J. y Stig, M. (2009). "Workaholism Among Norwegian Journalists: Gender Differences”. En: Equal Opportunities International, 28 (6), pp. 452-464.

Caro, F. J. y Díez, E. P. (2005). “Investigación cualitativa asistida por ordenador en economía de la empresa. En: Investigaciones europeas de dirección y economía de la empresa, 11 (2), pp. 45-58.

Carter, C. y Steiner, L. (eds.) (2004). Critical Readings: Media and Gender. Maidenhead: Open University Press.

Castellanos et al. (2010). "An Application of Grounded Theory to Cultural Tourism Research: Resident Attitudes to Tourism Activity in Santiponce”. En: Richards, G. (ed.). Cultural Tourism Research Methods (pp. 115-128). Oxfordshire, RU: Cabi Publishing. 
Correa, T. y Harp, D. (2011). "Women Matter In Newsrooms: How Power And Critical Mass Relate To The Coverage OfThe Hpv Vaccine”. En: Journalism \& Mass Communication Quarterly, 88 (2), pp. 301-319.

Craft, S. y Wanta, W. (2004). "Women in the newsroom: Influences of female editors and reporters on the news agenda”. En: Journalism \& Mass Communication Quarterly, 81 (1), pp. 124-138.

Djerf-Pierre, M. (2007). “The Gender of Journalism”. En: Nordicom Review, Jubilee Issue, pp. 81-104.

Ellingson, L. L. (2008). Engaging crystallization in qualitative research. Thousand Oaks, CA: Sage.

Glaser, B. y Strauss, A. (1967). The discovery of grounded theory: Strategies for qualitative research. Londres: Wiedenfeld and Nicholson.

Harp, D. (2007). Desperately Seeking Women Readers: U.S. Newspapers and the Construction of a Female Readership. Lanham: Lexington Books.

Kearney, M. C. (ed.) (2012). The Gender and Media Reader. Nueva York: Routledge.

Kim, K. H.y Yoon, Y. (2009). “The influence of journalists' gender on newspaper stories about women Cabinet members in South Korea”. En: Asian Journal of Communication, 19 (3), pp. 289-301

Krippendorff, K. (1990). Metodología de análisis de contenido. Teoría y práctica. Barcelona: Paidós Comunicación.

Len-Ríos, M., Rodgers, S., Thorson, E. y Yoon, D. (2005). "Representation of women in news and photos: Comparing content to perceptions". En: The Journal of Communication, 55, pp. 152-168.

Limor, Y. y Lavie, A. (2002). “The Feminization of The Media: The Case of Israel”. En: XXIII Annual Conference of the International Associa- 
tion for the Mass Communication Research (Iamcr), Gender and Communication Section. Barcelona, 21-26 de julio.

Melin-Higgins, M. (2001). "Coping With Journalism: Gendered Newsroom Culture”. En: Jornadas de Discusión sobre la Construcción de los Estereotipos de Género en los Medios de Comunicación. Facultat de Ciències de la Comunicació, UAB.

Murthy, D. y Anita, G. (2010). "Women in the Media and their Work Environment: A Report from Andhra Pradesh”. En: Indian Journal of Gender Studies, 17 (1), pp. 73-103.

Neveu, E. (2000). "Le Genre du Journalisme des Ambivalences de la Féminisation d'une Profesión”. En: Reveu Politix, 51, octubre. París: Publications Hermes.

Patton, M. (1990). Qualitative Evaluation and Research Methods. Newbury Park, CA: Sage.

Peters, B. (2001). Equality and Quality: Setting Standards for Women in Journalism. Bruselas: IFJ.

Rodgers, S. y Thorson, E. (2003). "A socialization perspective on male and female reporting". En: Journal of Communication, 53 (4), pp. 658-675.

Stempel, G. H. y Westley, B. H. (eds.) (1989). Research Methods in Mass Communication, NJ: Prentice Hall, Englewood Cliffs.

Strauss, A. y Corbin, J. (2002). Bases de la investigación cualitativa. Técnicas y procedimientos para desarrollar la teoría fundamentada. Medellín: Universidad de Antioquia.

Tracy, S. J. (2010). “Qualitative Quality: Eight 'Big-Tent' Criteria for Excellent Qualitative Research. Qualitative Inquiry, 16, pp. 837-851. 
Van Zoonen, L. (1994). Feminist Media Studies. Londres: Sage.

Weick, K. E. (2007). The generative properties of richness. Academy of Management Journal, 50, pp. 14-19.

Winter, G. (2000). "A comparative discussion of the notion of validity in qualitative and quantitative research". En: Qualitative Report, 4 (3 y4). Disponible en: http://www.nova.edu/ssss/QR/QR4-3/winter.html [fecha de consulta: 15 de octubre de 2011].

Ziamou, T. (2000). Women Make The News: A Crack In The "Glass Ceiling"? Unesco.

Zoch, L. M. y Turk, J. V. (1998). "Women making news: Gender as a variable in source selection and use". Journalism \& Mass Communication Quarterly, 75 (4), pp. 762-775

Yin, R. (1984). Case Study Research. Beverly Hills, CA: Sage.

Kinnebrock, S. (2009). "Revisiting journalism as a profession in the 19(th) century: Empirical findings on women journalists in Central Europe". En: Communications-European Journal of Communication Research. 